\title{
COMMENTARY
}

\section{Layer-by-layer deposition of nanoscale structures}

\author{
Abhishek Kumar and Jayant Kumar \\ Center for Advanced Materials, Department of Physics, University of Massachusetts Lowell, \\ Lowell, MA 01854, USA \\ Jayant_kumar@uml.edu
}

Fabrication of ultrathin films of polymers or polymer nanocomposites with precise control of thickness and composition is of great interest due to a variety of possible applications for optics and electronics. Several techniques have been reported in the last few decades, amongst which are the Langmuir-Blodgett (LB) technique, self-assembled monolayer (SAM) technique, and the layer-by-layer adsorption $(\mathrm{LbL})$ of materials. Both the LB and the SAM techniques are capable of assembling small organic molecules, polymers, proteins and nanoparticles for optical and electronic devices as well as surface modification. Both techniques provide ultrathin films of controlled thickness, composition and orientation. The LB technique requires expensive instrumentation and is a laborious process. One of the limitations of SAM assembly is the requirement of very specific substrates such as noble metals for thiols, or silica or metal oxides for silanes.

A versatile technique for deposition of ultrathin films is the LbL assembly of materials, as demonstrated by Decher and co-workers [1]. In the last two decades, this technique has been widely adopted for the fabrication of nanostructures with tailored properties. The LbL technique is attractive due to its simplicity and versatility. In most cases, the deposition can be carried out from aqueous solution of molecules and polymers or suspensions of nanoparticles on the bench top. LbL technique can be extended to create conformal coatings on non-planar geometries including porous membranes, colloidal particles, fibers, living cells, etc [2-4].

In general we can classify LbL techniques by the kind of interactions which dominate the assembly process. Accordingly, the following distinct classes can be identified: (1) electrostatically assembled multilayers from highly charged polycations, polyanions or charged nanoparticles; (2) electrostatically assembled multilayers from partially charged polyelectrolytes; (3) LbL multilayers where interactions such as hydrogen bonding play an important role in adsorption; and (4) LbL multilayers produced via specific interactions, such as biotin-avidin affinity binding. With the appropriate choice of these interactions, the thickness and morphology of the films can be effectively controlled.

In the electrostatic LbL process, spontaneous sequential adsorption of oppositely charged materials is carried out from dilute aqueous solutions on a charged substrate. During the deposition process, an oppositely charged substrate is dipped in a solution of either a cationic or an anionic polyelectrolyte (molecules or nanoparticles) for a certain period of time. If the concentration of the charged species (i.e., polyelectrolyte) is sufficient, adsorption occurs until there is a complete charge reversal at the surface. The process is then repeated with a solution of either an anionic or a cationic polyelectrolyte.

Figure 1(a) shows a schematic diagram of the experimental procedure of adsorption in LbL films. After adsorption of each layer, a simple washing step is performed to remove weakly or nonspecifically bound polymers, molecules or particles adhering to the substrate. Moreover, this step also prevents the contamination of the solution in the next dipping 
process. Automated systems can put together to facilitate the repetitive dipping steps for multilayer deposition. Iterating the dipping cycles with alternating adsorption of polyanions and polycations results in the stepwise growth of an ultrathin film. Since the process only involves adsorption from solutions, in principle there are no restrictions with respect to substrate size and shape. In most cases, the LbL deposition technique leads to a highly interpenetrating structure of the cationic and anionic species, thereby yielding amorphous films. However, in some cases appropriate adsorption conditions can also lead to orientational order.

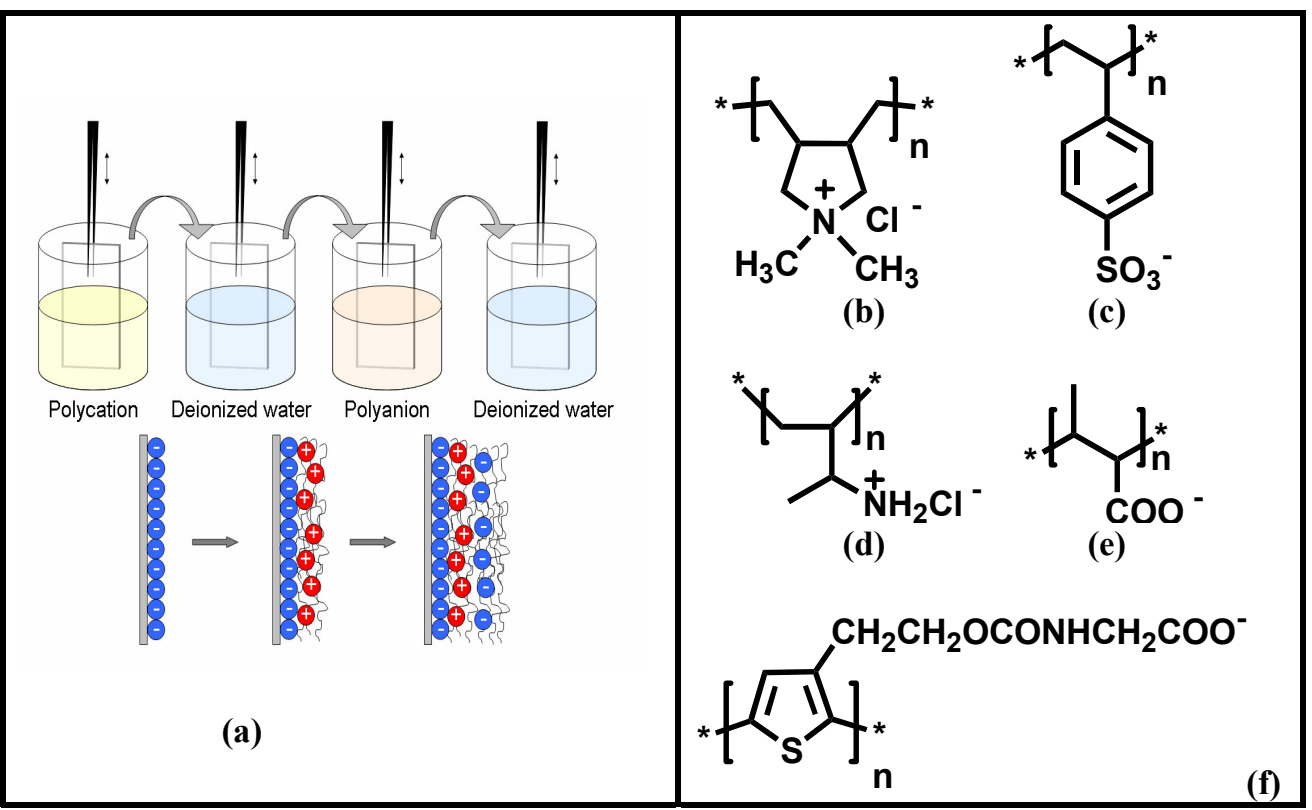

Fig. 1. (a) Schematic of LbL assembly process, and chemical formulas of (b) PDAC, (c) PSS, (d) PAH, (e) PAA and (f) H-PURET.

The majority of LbL films investigated in early years were made from highly charged polyelectrolytes or strong polyelectrolytes such as (i) Poly (diallyldimethylammonium chloride), PDAC (Fig. 1(b)), which is a commonly used strong cationic polyelectrolyte, and (ii) Polystyrene sulfonate (PSS) (Fig. 1(c)), which is an example of strong anionic electrolyte. The degree of ionization of the strong polyelectrolytes does not change significantly with the change in $\mathrm{pH}$ of the solution. Many weak polyelectrolytes have also been extensively used. These include (i) Poly (allylamine hydrochloride), PAH (Fig. 1(d)), which is an example of a weak cationic polyelectrolyte, and (ii) Poly acrylic acid (PAA), which is a weak anionic polyelectrolyte (Fig. 1(e)). In weak polyelectrolytes, the degree of ionization varies considerably with the change in $\mathrm{pH}$. When a weak polyelectrolyte is used, the thickness of the adsorbed material can be varied by an order of magnitude, depending on the experimental conditions such as $\mathrm{pH}$ and the ionic strength.

Figure 1(f) shows the structure of a polyanion, hydrolyzed poly[2-(3-thienyl)ethanol butoxy carbonyl-methyl urethane] (H-PURET), which is a semiconducting polymer based on a functionalized thiophene monomer. It has been used extensively by our group for making optical sensors. In the unionized form, polymers containing unionized acid and/or amine groups may be adsorbed utilizing hydrogen-bonded interactions.

The LbL technique can be monitored and characterized by optical, electrical and surface characterization techniques such as atomic force microscopy (AFM) and scanning tunneling 
microscopy (STM). UV-Visible absorption spectroscopy and ellipsometery are commonly used to monitor the thickness of the layers.

The measured peak absorption of a single bilayer is shown in Fig. 2(left) as a function of dipping time. The absorbance in this case saturates at a dipping time about 5 min. Figure 2(middle) shows the UV-Visible absorption spectra for the multilayers prepared by sequential deposition of PDAC/H-PURET bilayers. Importantly, as shown in Fig. 2(right), the peak absorption changes linearly with deposition cycles in a multilayer deposition process, which signifies that the amount of material deposited for each layer is the same, and also confirms the regular stepwise growth of the film.

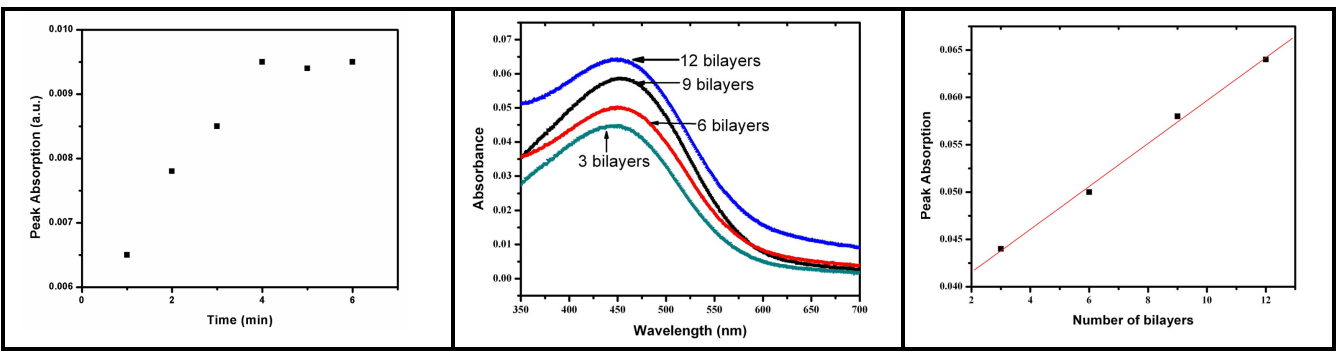

Fig. 2. (Left) Peak absorption of one bi-layer plotted against adsorption time, (middle) absorbance spectra of PDAC/H-PURET multilayers, and (right) peak absorption of PDAC/H-PURET with number of multilayers.

The technique of layer-by-layer adsorption is widely used to immobilize photoluminescent indicators in polymer thin films as well as on the surfaces of electrospun nano fibers for the fabrication of fluorescent chemical sensors [5]. The technique is also exploited to make a diverse array of nanostructured materials such as freestanding submicron thin films, nanotubes, and hollow capsules [6]. It is also extensively used to fabricate films containing nanoparticles. By taking advantage of the charges on nanoparticles, the LbL technique has been used to fabricate surfaces exhibiting either superhydrophobic or superhydrophilic behavior [7].

The simplicity of the LbL technique makes it attractive for potential applications in many areas of technological importance. Nucleic acid, proteins and viruses have been successfully used as components to prepare LbL films [3]. The technique has been used to fabricate oriented multilayer films of photoactive proteins [8] as well as to fabricate organic lightemitting diodes, electrochromic devices, and photovoltaic devices $[9,10]$.

In conclusion, the LbL technique over the last two decades has become extremely popular to create a wide variety of nanostructures and multilayer thin films with controlled morphology and composition. We expect that the LbL technique will continue to find applications in diverse areas such as medicine, surface modifications as well as optoelectronic devices.

\section{Acknowledgement}

Funding from the US Army Research Lab, US Army Natick Soldier Research Center and National Science Foundation is gratefully acknowledged. We thank past and present members of our group at the Center for Advanced Materials, UMass Lowell. 


\section{References}

[1] G. Decher, J. D. Hong, and J. Schmitt, "Buildup of ultrathin multilayer films by a self-assembly process: III. Consecutively alternating adsorption of anionic and cationic polyelectrolytes on charged surfaces," Thin Solid Films 210/211, 831-835 (1992) [doi: 10.1016/0040-6090(92)90417-A].

[2] S. K. Tripathy, J. Kumar, and H. S. Nalwa, Eds., Handbook of Polyelectrolytes and Their Applications, Vols. 1, 2 \& 3, American Scientific Publishers, Los Angeles, CA, USA (2000).

[3] P. T. Hammond, "Form and function in multilayer assembly: New applications at the nanoscale," Adv. Mater. 16, 1271-1293 (2004) [doi: 10.1002/adma.200400760].

[4] Z. Tang, Y. Wang, P. Podsiadlo, and N. A. Kotov, "Biomedical applications of layer-by-layer assembly: From biomimetics to tissue engineering," Adv. Mater. 18, 3203-3224 (2006) [doi: 10.1002/adma.200600113].

[5] X. Wang, Y.-G. Kim, C. Drew, B.-C. Ku, J. Kumar, and L. A. Samuelson, "Electrostatic assembly of conjugated polymer thin layers on electrospun nanofibrous membranes for biosensors," Nano Lett. 4, 331-334 (2004) [doi: $10.1021 / \mathrm{nl} 034885 \mathrm{z}]$.

[6] Y. Wang, A. S. Angelatos, and F. Caruso, "Template synthesis of nanostructured materials via layer-by-layer assembly," Chem. Mater. 20, 848-858 (2008) [doi: $10.1021 / \mathrm{cm} 7024813]$.

[7] L. Zhai, F. C. Cebeci, R. E. Cohen, and M. F. Rubner, "Stable superhydrophobic coatings from polyelectrolyte multilayers," Nano Lett. 4, 1349-1353 (2004) [doi: 10.1021/n1049463j].

[8] J.-A. He, L. Samuelson, L. Li, J. Kumar, and S. K. Tripathy, "Oriented bacteriorhodopsin/polycation multilayers by electrostatic layer-by-layer assembly," Langmuir 14, 1674-1679 (1998) [doi: 10.1021/la971336y].

[9] V. Jain, M. Khiterer, R. Montazami, H. M. Yochum, K. J. Shea, and J. R. Heflin, "High-contrast solid-state electrochromic devices of viologen-bridged polysilsesquioxane nanoparticles fabricated by layer-by-layer assembly," ACS App. Mater. Interf. 1, 83-89 (2009) [doi: 10.1021/am8000264].

[10] J.-A. He, R. Mosurkal, L. A. Samuelson, L. Li, and J. Kumar, "Dye-sensitized solar cell fabrication by electrostatic layer-by-layer assembly of amphoteric $\mathrm{TiO}_{2}$ nanoparticles," Langmuir 19, 2169-2173 (2003) [doi: 10.1021/la020639b]. 